\title{
Cytogenetic analysis using telemedicine consultation: An improved means of providing expert cross-coverage
}

\author{
Jeanne M. Meck, $P h D^{1}$, Gaurang Munshi ${ }^{2}$, Judy Plempel ${ }^{2}$, Stephen Amato, MD ${ }^{3}$, and Christian Macedonia, $M D^{1}$
}

\begin{abstract}
Purpose: We used telemedicine in providing cross-coverage for a clinical cytogenetics laboratory. A genetic teleconsultation system was used to provide expert cross-coverage for a laboratory in a neighboring metropolitan region for a 6 month period while the usual provider of these services was on military reserve duty. Methods: The teleconsultation system was a commercially available Perceptive Scientific Instruments (PSI) workstation. Five hundred thirty-nine cytogenetic cases were performed during the study period in the home laboratory in Baltimore, Maryland. Results: Karyotypes and supporting metaphase spreads were transmitted by modem to the covering director, whereas work sheets and reports were faxed. Physical transfer of data was not necessary, and turn-around-time was not increased. Conclusion: This ability to employ a remote part time director has significant benefits for the laboratory with an absentee director for short or even extended periods of time. We conclude that the use of telemedicine in clinical cytogenetics proved to be an efficient and a cost effective means of providing expert genetics services to a region during a time of cross-coverage need. Genetics in Medicine, 1999:1(7):
\end{abstract} 328-331.

Key Words: Cytogenetics, telemedicine, computers in medicine

Telemedicine is the science or practice of the diagnosis, treatment, and prevention of disease performed by individuals located at a point distant from the patient. The modern definition also implies that some sort of telecommunications equipment and/or computer technology is used in the process. ${ }^{1.2}$ Telemedicine technology has matured primarily under test conditions established by organizations like the US military, ${ }^{3}$ NASA, and the Indian Health Service. ${ }^{4}$ However, it has also grown dramatically in importance in more traditional medical environments. The progress in telemedicine has accelerated in the past few years as current health care policies have limited the availability of specialists and decreased the budget for medical testing and interpretation. Recently, telemedicine has been used internationally in cytology for the purposes of diagnosis, consultation, and education. ${ }^{5.6}$ Similarly, cytogenetic consultations over long distances can be conducted using the newest desktop computing systems with image processing and network upgrades.

Although telemedicine has been described in many medical disciplines, to our knowledge, this is the first report of use of

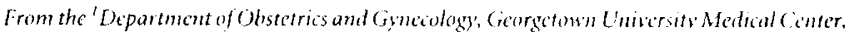

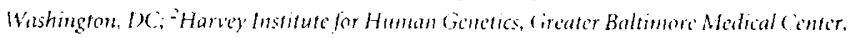

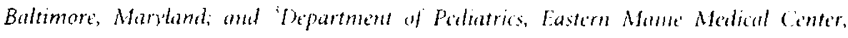
Rangror, Maine:

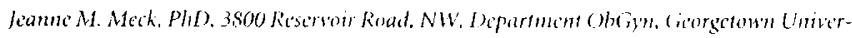
sity Medical Center, Washington. DC $20(1)$.

Recoved: September 13, 1999

Aicepted: October 25, 1999. this technology for cytogenetics cross-coverage. ${ }^{7}$ In this report we demonstrate how imaging systems that are now commonly used in clinical cytogenetics laboratories for capturing, analyzing, and archiving metaphase images can be used to provide expert cross-coverage in the absence of a laboratory director. From January through June 1997, the Greater Baltimore Medical Center had an unexpected absence of the cytogenetics director because he was called for a 6 month tour of active duty from the US Army reserve. The Baltimore laboratory staff was highly experienced and could perform daily laboratory functions such as tissue culture, chromosome preparation and analysis, image capturing, and karyotyping. However, a board certified cytogeneticist was needed to review and sign cases on a daily basis. Hiring an interim director would have been both impractical and costly. On-site review of cases on a daily basis by a local director would be difficult. Therefore, remote review of cases by a laboratory director based in Washington, DC was established via a telemedicine link.

\section{MATERIALS AND METHODS}

The teleconsultation system was a commercially available Perceptive Scientific Instruments (PSI) workstation (League City, TX) (Fig. 1). It consisted of a Macintosh PowerPC with an $80 \mathrm{MHz}$ 601RISC processor, $32 \mathrm{Mb}$ of RAM, and $2 \mathrm{Mb}$ of VideoRAM. It had a 15 inch SVGA display for soft copy interpretation. Hard copy was stored at the Baltimore facility. Images were acquired with a 3 chip $1 / 2$ inch charge-coupled device (CCD) came in a proprietary image format at 2-4 Mbytes. 

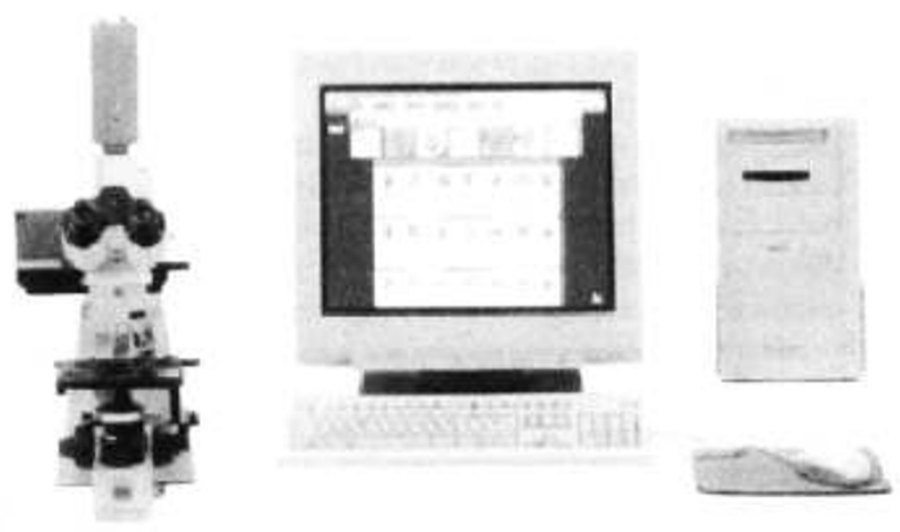

Fig. 1 The teleconsultation system that was used to capture metaphase images and karyotypes included a photomicroscope, CCD camera, and a Macintosh PowerPC with Perceptive Scientific Instruments software.

They were subsequently compressed at a 10:1 compression ratio using standard algorithms similar to the JPEG and TIFF routines for image transfer. Display and image transfer soft- ware were written by Perceptive Scientific Instruments Inc. and designed specifically for cytogenetics consultation.

Five hundred thirty-nine cytogenetic cases were performed during the study period in the home laboratory in Baltimore. Metaphases were imaged and karyotyped as usual on a PSI imaging system and transmitted by an analog modem operating at 56 kilobits per second to the off-site director. All karyotypes and metaphases were reviewed on a Macintosh Performa 6400 computer by the off-site director using a soft copy display at a screen resolution of $800 \times 600$ pixels at 36 bits per pixel. Images were transferred in JPEG format (Fig. 2).

Karyotype score sheets, request forms with pertinent clinical information, and typed laboratory reports were faxed for supporting documentation. Typed reports, which had been written by the laboratory supervisor, were faxed to the off-site director for approval. These were either signed "as is" and faxed back to the laboratory or corrections were made by telephone or fax.

\section{RESULTS}

The types of cases were varied and represented the full range seen for cytogenetic analysis including amniotic fluid, chori-

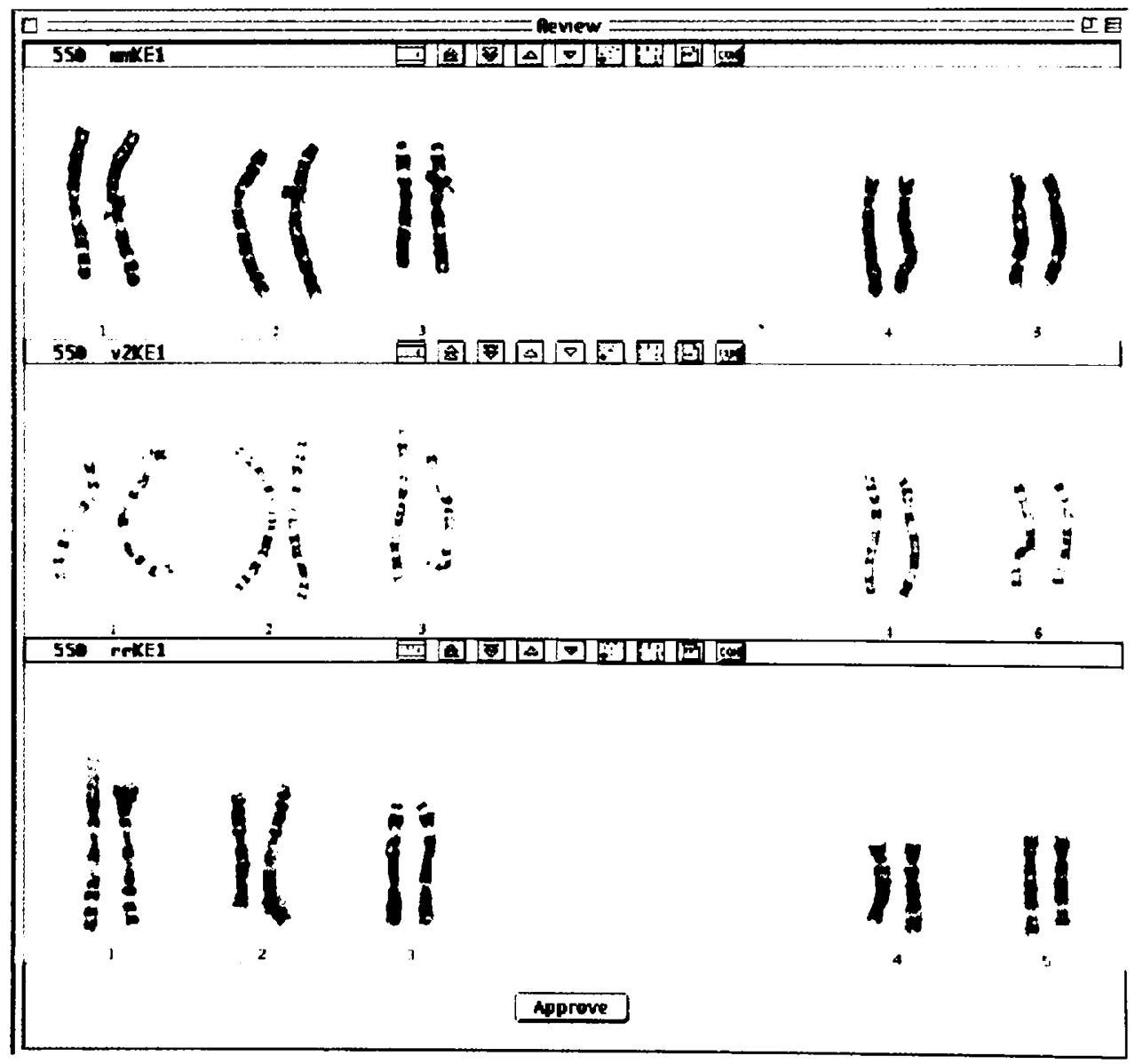

Fig. 2 Karyotype image display for the off-site director's review. One line of the karyotype from multiple metaphases on the patient can be viewed simultaneously. 
onic villus sampling (CVS), peripheral blood, bone marrows, and products of conception (Table 1). Cases were reviewed by the off-site director 4-5 days per week so that the turn-aroundtime was not adversely affected in the absence of on-site review. The average turn-around-time for all specimen types for the 6 month period during the time of remote review (TAT $=8.3$ days, $\mathrm{N}=539$ cases) was not increased over the preceding 6 months of standard on-site review (TAT $=8.4$ days, $\mathrm{N}=626$ cases) (Table 1).

Image quality of the karyotypes after file conversion, compression, transmission, and decoding was equivalent to that observed by the technologists on-site. This was evaluated by comparison of the technologists' estimate of the number of G-bands per haploid set of chromosomes, which was submitted to the reviewing director by fax and was rarely different from the reviewing director's estimate. The majority of cases were in the $400-550$ band range. None of these cases required the physical transfer of laboratory materials or personnel.

There was a total of 82 abnormal cases during the period of telemedicine review yielding an abnormality rate of $15.21 \%$ for all specimen types, compared to $15.81 \%$ (99 of 261) for the previous 6 month period of standard review. The abnormalities found were both numerical and structural and included trisomies, monosomy $\mathrm{X}$, reciprocal and robertsonian translocations, pericentric and paracentric inversions, and deletions. If there was any difficulty in making a diagnosis from the submitted karyotypes, the off-site director would request additional karyotypes from the laboratory, which would be submitted via modem the following day. Phone calls were made by the off-site director to the referring physician on all the abnormal amniotic fluid cases $(\mathrm{N}=13)$ and abnormal CVS cases $(\mathrm{N}=$ 2). Abnormal reports for other types of specimens were transmitted by fax to the referring physician and they were given the option to telephone the off-site director to discuss the significance of the abnormalities.

\section{DISCUSSION}

There are a number of reasons why the role of telemedicine in cytogenetics is both timely and important: (1) Institutions have fewer resources that they can commit to cytogenetics; (2)
Laboratory directors have increasing obligations off-site requiring the ability to review cases remotely; (3) Improvement in cytogenetic imaging systems and telemedicine links has been dramatic in the past decade.

During the 6 month period that this report covers, there was no increase in laboratory turn-around-time, nor was there a break in access to a clinical cytogeneticist. Referring physicians did not need to change their referral patterns for cytogenetic analysis. They received a laboratory report with which they were familiar and could readily interpret. The off-site covering director was readily available to both the referring physicians and the laboratory staff for telephone consultations. The cost of hiring a part-time director and purchasing the necessary computer hardware and software was minimal compared with hiring a full time on-site director or a local director who would make daily visits to the laboratory. These cost savings for a 6 month period are estimated to have been approximately $\$ 25,000$.

With respect to medical-legal issues, the use of telemedicine is similar to the use of an out-of-state laboratory for chromosome analysis. Transporting specimens across state lines to a commercial or specialized academic laboratory for genetic analysis is a common practice. According to the Standards and Guidelines of the American College of Medical Genetics (ACMG), a Cytogenetics Laboratory Director must have an appropriate doctoral degree, at least 2 years of postdoctoral training or experience in cytogenetics, and be boarded by either the American Board of Medical Genetics or the Canadian College of Medical Genetics in Clinical Cytogenetics. ${ }^{8}$ These standards should also apply to a remote reviewer, although that issue is not specifically addressed. Laboratory directors are required by the Clinical Laboratory Amendment (CLIA) to be "accessible to the laboratory to provide on-site, telephone or electronic consultation as needed." 9 In a case such as this, in which a director is called for military duty, the absentee director can delegate responsibility to another qualified director who, in turn, needs to be accessible. There is no qualification in CLIA 88 regarding the location of the interim director. However, according to the Standards and Guidelines of the ACMG, "a laboratory director must be on-site regularly (at least weekly)... " ${ }^{8}$ Of course, an on-site director should be the standard

Table 1

Type, number, and turn-around time of samples analyzed using telemedicine versus standard on-site review

\begin{tabular}{|c|c|c|c|c|}
\hline Type of Specimen & $\begin{array}{l}\text { Sample Number } \\
\text { during } 6 \text { mo. } \\
\text { telemedicine trial }\end{array}$ & $\begin{array}{l}\text { Average TAT using } \\
\text { telemedicine } \\
\text { (in days) }\end{array}$ & $\begin{array}{l}\text { Sample Number } \\
\text { during } 6 \text { mo. } \\
\text { standard review }\end{array}$ & $\begin{array}{l}\text { Average TAT using } \\
\text { standard review } \\
\text { (in days) }\end{array}$ \\
\hline Amniotic fluid & 323 & 8.4 & 363 & 8.5 \\
\hline Peripheral blood & 42 & 5.9 & 58 & 6.1 \\
\hline Bone marrow & 28 & 3.8 & 31 & 4.0 \\
\hline Total/Average & 539 & 8.3 & 626 & 8.4 \\
\hline
\end{tabular}

TAT, turn-around time. 
for all clinical laboratories to ensure that laboratory regulations and quality assurance programs, as well as laboratory staff education, are fully carried out. Nevertheless, there are times when a director, either for personal or professional reasons, must be away from the laboratory for an extended period of time and, currently, the ACMG guidelines do not address this issue.

There are numerous other potential applications of telemedicine in cytogenetics. Directors who are off-site for professional meetings can access their karyotype images from lap top computers. Telemedicine can also be used for multi-expert review of complex or ambiguous cases, which enhances collaboration among peers and is also important for laboratories in remote locations or with few experienced local cytogeneticists. The educational potential of telemedicine is also intriguing and can assist in continuing education for trained technologists and directors, competency testing for new technologists, and national proficiency testing programs for cytogenetics laboratories.

Telemedicine works best when it is applied to clinical problems requiring high levels of expertise and where these problems can be addressed by review of largely visual data. Furthermore, it is most optimally used when there is a gap in the physical distribution of the expert consultants. We conclude that cytogenetics cross-coverage fits well into this model. We anticipate that the majority of cytogenetics laboratories will use telemedicine as an adjunct to their expanding role in the medical community.

\section{Acknowledgment}

The authors would like to thank Perceptive Scientific Instruments for their assistance in providing the graphic for this manuscript.

\section{References}

1. Scannell KM, Perednia DA, Kissman HM. Telemedicine: Past, present, future (CBM 95-4), U.S. Dept of Health and Human Services, Public Health Service, National Institutes of Health, National Library of Medicine, Reference Section. Pittsburgh, PA, 1995;1.

2. Office of Rural Health Policy, Department of Health, and Human Services Report. Exploratory evaluation of rural applications of telemedicine. Feb. 1997.

3. Institute of Medicine. Telemedicine: A guide to assessing telecommunications in health care. National Academy Press, 1996.

4. Fuchs M. Provider attitudes toward STARPAHC, a telemedicine project on the Papago Reservation. Med Care 1979;17:59-68.

5. Galvez J, Howell L, Costa MJ, Davis R. Diagnostic concordance of telecytology and conventional cytology for evaluating breast aspirates. Acta Cytol 1998;42:663-637.

6. Marsan C, Vacher-Lavenu MC. Telepathology: A tool to aid in diagnosis and quality assurance in cervicovaginal cytology. Cytopathology 1995;6:339-342.

7. Perdina D, Allen A. Telemedicine technology and clinical applications. JAMA 1995; 273:483-488.

8. Standards and Guidelines. Clin Genet Laboratories. The American College of Medical Genetics, Laboratory Practice Committee. 1993;2.

9. Clinical Laboratory Amendment of 1998 (CLIA 88). Department of Health and Human Services, Health Care Financing Administration. Standard 493.1445, 1988. 